\title{
Impacto de uma intervenção no estigma em saúde mental e ansiedade intergrupal
}

Impact of an intervention on stigma in mental health and intergroup anxiety Impacto de una intervención sobre el estigma en salud mental y ansiedad intergrupal

\author{
Ana Isabel Fernandes Querido ${ }^{1}$ ib https://orcid.org/0000-0002-5021-773X \\ Catarina Cardoso Tomás ${ }^{1}$ ib https://orcid.org/0000-0003-3713-3352 \\ Daniel Ricardo Simões de Carvalho ${ }^{1}$ io https://orcid.org//0000-0002-5058-525X \\ João Manuel Ferreira Gomes ${ }^{1}$ ic https://orcid.org/0000-0002-4459-4950 \\ Marina Soffia Silva Cordeiro' io hitps:///orcid.org/0000-0002-5953-1565
}

\section{Como citar:}

Querido Al, Tomás CC, Carvalho DR, Gomes JM, Cordeiro MS. Impacto de uma intervenção no estigma em saúde mental e ansiedade intergrupal. Acta Paul Enferm. 2020;33:APE20190226.

DOI

http://dx.doi.org/10.37689/actaape/2020A00226

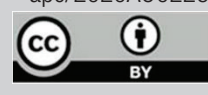

Descritores

Saúde mental; Transtornos mentais

Keywords Social stigma; Anxiety; Mental health; Mental disorders

Descriptores

Estigma social; Ansiedad; Salud mental; Transtornos mentales

\section{Submetido}

2 de Agosto de 2018

Aceito

7 de Outubro de 2019

Autor correspondente Ana Isabel Fernandes Querido Email: ana.querido@ipleiria.pt

\section{Resumo}

Objetivo: Avaliar a eficácia de uma intervenção na redução das atitudes estigmatizantes e ansiedade perante 0 doente mental em estudantes de enfermagem.

Métodos: Estudo quase experimental, realizado com uma amostra intencional de 99 estudantes de enfermagem de um instituto universitário da região centro de Portugal. No grupo de controlo, os estudantes desenvolveram o plano de ensino clínico em Enfermagem de saúde mental e psiquiátrica em serviços de psiquiatria, conforme o plano de estudos ( $n=50$ ). No grupo de teste, além de cumprirem o plano de estudos, os estudantes participaram de um programa psicoeducativo dirigido ao estigma $(n=49)$. Utilizaram-se as versões portuguesas da Mental IIIness: Clinicians' Attitudes Scale (MICA-4) e AQ27 para avaliação do estigma e a Intergroup Anxiety Scale (EAl) para medir a ansiedade intergrupal.

Resultados: A amostra apresenta homogeneidade de variáveis no momento inicial $(p>0,05)$, com melhorias estatisticamente significativas das atitudes estigmatizantes e redução da ansiedade perante 0 doente mental em ambos os grupos $(p<0,005)$. 0 impacto da intervenção não foi significativo, tendo 0 grupo de teste melhorado as atitudes estigmatizantes de medo $(p=0,03)$ e piorado relativamente à ajuda $(p=0,04)$.

Conclusão: Verificou-se um impacto positivo do ensino clínico na redução da ansiedade perante o doente mental, conduzindo a uma redução do estigma em saúde mental. A intervenção dirigida ao estigma não revelou efeitos significativos no estigma global nem na ansiedade intergrupal o que aponta para a necessidade futura da sua reformulação e comparação com amostras de estudantes de outras instituições de ensino de enfermagem.

\section{Abstract}

Objective: To assess the effectiveness of an intervention to reduce stigmatizing attitudes and anxiety towards people with mental illness among nursing students.

Methods: This was a quasi-experimental study including an intentional sample of 99 nursing students from university of Central Portugal. In the control group, students developed the clinical teaching plan in mental health and psychiatric nursing in psychiatric health services, according to the syllabus $(n=50)$. In the experimental group, in addition to complying with the syllabus, the students participated in a psychoeducational program oriented towards stigma ( $\mathrm{n}=49)$. To assess stigma, the Portuguese versions of Mental IIIness: Clinicians' Attitudes Scale (MICA-4) and AQ27 were used; and to measure intergroup anxiety, the Intergroup Anxiety Scale (IAS).

Results: The sample presents homogeneous variables in the initial time ( $p>0.05)$, with statistically significant improvement of stigmatizing attitudes and reduction of anxiety towards people with mental illness in both studied groups $(p<0.005)$. The impact of the intervention was not significant, the experimental group improved 
stigmatizing attitudes regarding fear $(p=0.03)$ and the ones regarding help had worsened $(p=0.04)$.

Conclusion: We verified a positive impact of clinical teaching in the reduction of anxiety towards people with mental illness, which led to a reduction of stigma associated to mental health. The intervention oriented towards stigma did not reveal significant effects concerning global stigma, nor intergroup anxiety, which showed the future necessity of its reformulation and comparison with samples of students from other nursing universities.

\section{Resumen}

Objetivo: Evaluar la eficacia de una intervención en la reducción de actitudes estigmatizantes y ansiedad ante la persona con enfernedad mental en estudiantes de Enfermería.

Métodos: Se trata de un estudio cuasi experimental, realizado con un muestreo intencional de 99 estudiantes de Enfermería de un instituto universitario de la región centro de Portugal. En el grupo de control, los estudiantes realizaron el plan de enseñanza clínica en enfermería de salud mental y psiquiátrica en servicios de psiquiatría, de acuerdo con el plan de estudios $(n=50)$. En el grupo experimental, además de cumplir con el plan de estudios, los estudiantes participaron en un programa psicoeducativo orientado al estigma ( $\mathrm{n}=49$ ). Se utilizaron las versiones portuguesas de la Mental IIIness: Clinicians' Attitudes Scale (MICA-4) y AQ27 para evaluar el estigma y la Intergroup Anxiety Scale (EAl) para medir la ansiedad intergrupal.

Resultados: La muestra presentó homogeneidad de variables en el momento inicial ( $p>0,05)$, con mejoras estadísticamente significativas de las actitudes estigmatizantes y reducción de la ansiedad ante la persona con enfermedad mental en ambos grupos ( $p>0,005)$. El impacto de la intervención no fue significativo: el grupo experimental mejoró las actitudes estigmatizantes de miedo $(p=0,03)$ y empeoró relativamente la ayuda $(p=0,04)$.

Conclusión: Se verificó un impacto positivo de la enseñanza clínica en la reducción de la ansiedad ante la persona con enfermedad mental, lo que lleva a una reducción del estigma en salud mental. La intervención orientada al estigma no reveló efectos significativos en el estigma global ni en la ansiedad intergrupal, lo que advierte la necesidad futura de su reformulación y comparación con muestras de estudiantes de otras instituciones educativas de Enfermería.

\section{Introdução}

O estigma face à doença mental continua a ser uma realidade presente com consequências para todos os envolvidos, quer sejam os doentes, famílias ou profissionais de saúde. Existe uma forte relação entre a redução do estigma e a promoção de atitudes positivas face à pessoa com doença mental. ${ }^{(1)}$ Nos profissionais de saúde este fenómeno constitui um problema uma vez que as suas atitudes estigmatizantes, muitas vezes baseadas em crenças e mitos disseminadas e enraizados, têm um impacto superior ao da população geral, ${ }^{(2,3)}$ representam uma barreira importante no acesso a cuidados de saúde mental e afetam a perceção e aceitação da doença. ${ }^{(4-6)}$

Os estudantes de enfermagem demonstram por vezes atitudes negativas e pouco conhecimento face à doença mental, que se mantêm mesmo após o terminus da sua formação, com impacto na sua futura prática profissional. ${ }^{(2)}$ Os planos de estudos em enfermagem deveriam incluir programas de consciencialização para o estigma e suas consequências, uma vez que este período é adequado para a mudança de atitudes face ao estigma da doença mental. ${ }^{(2,7,8)}$

As atitudes estigmatizantes dos estudantes tendem a diminuir através da interação com profissionais e serviços de saúde mental. ${ }^{(4)}$ Diversos autores referem a educação e o contacto como duas das estratégias mais eficazes no combate ao estigma. ${ }^{(4,5,8-10)}$
Várias intervenções têm sido realizadas associando a educação e o contacto, obtendo reasultados ao nível da melhoria das atitudes estigmatizantes face à doença mental, especialmente se houver alguma integração entre ambas as estratégias, apostando na autoreflexão e desenvolvimento pessoal. ${ }^{(8,11-13,14)}$

A componente educativa deste tipo de intervençóes torna-se mais eficaz se as informaçóes transmitidas forem o mais factuais possíveis, recorrendo a estratégias educativas interessantes claras e simples. ${ }^{(12)}$ A eficácia do contacto depende da sua qualidade e de fatores como carga emocional, atitudes face ao estigma e o acompanhamento efetuado durante o mesmo, ${ }^{(10)}$ sendo fundamental a atitude dos enfermeiros durante o contacto com pessoas com doença mental quando desempenham o papel de supervisores de estudantes de enfermagem. ${ }^{(13)}$

Uma das emoçóes descritas pelos estudantes antes de iniciarem o ensino clínico na área da saúde mental é a ansiedade, que em conjunto com o medo, inquietação e a rejeição estão relacionadas em parte com mitos, estereótipos e atitudes negativas e ignorância ou ideias preconcebidas sobre possíveis episódios de agressividade com que se podem vir a deparar. ${ }^{(13,15)}$ Como forma de atenuar ou combater estas emoçóes torna-se importante que os estudantes tenham contato prévio com a atividade profissional e algum treino e conhecimento específico sobre esta temática, sobretudo em contexto de ensino clínico. ${ }^{(15)}$ 
Sáo escassos os estudos realizados em Portugal sobre o impacto de intervençóes dirigidas ao estigma em contextos clínicos, apesar da evidência da presença de estigma e de emoçóes negativas como a ansiedade entre os estudantes. ${ }^{(1,14-17)}$ Algumas experiências no Reino Unido demonstram a eficácia de intervençôes dirigidas ao estigma como a Responding to Experienced and Anticipated Discrimination (READ training) na redução da ansiedade e das atitudes estigmatizantes em contextos clínicos com estudantes de Medicina. ${ }^{(16)}$

Com este estudo pretendeu-se avaliar a eficácia de uma intervenção na redução das atitudes estigmatizantes e ansiedade perante o doente mental em estudantes de enfermagem. Colocámos como hipóteses que: (H1) Há uma redução significativa no nível de atitudes estigmatizantes e do nível de ansiedade intergrupal dos estudantes entre o primeiro e o segundo momento de avaliação; (H2) Há diferenças significativas na redução do nível de estigma e de ansiedade intergrupal nos estudantes do grupo de teste comparativamente aos do grupo de controlo.

\section{Métodos}

Trata-se de um estudo quase experimental do tipo antes e depois, desenvolvido com estudantes de enfermagem do terceiro ano do curso de licenciatura, a realizar ensino clínico de Enfermagem de saúde mental e psiquiátrica em serviços de internamento de agudos em hospitais da regiáo centro de Portugal, entre março de 2016 e junho de 2019.

Foram elegíveis para o estudo os estudantes matriculados no ensino clínico de enfermagem de saúde mental e psiquiátrica da instituição de ensino superior de saúde onde decorreu o estudo no período estipulado $(n=165)$. Incluíram-se estudantes maiores de 18 anos, com nacionalidade portuguesa e que aceitassem livremente participar na pesquisa. Foram excluídos os estudantes em programas de mobilidade e intercâmbio nacional ou internacional. Todos os estudantes que aceitaram participar da pesquisa assinaram o termo de consentimento livre e esclarecido. $\mathrm{O}$ recrutamento decorreu de forma faseada em cada ano curricular, antes da realização do ensino clínico.
O estudo foi desenvolvido nos hospitais onde decorreu o ensino clínico e cumpriu as normas de ética em pesquisa, tendo sido aprovado pela Comissão Nacional de Proteção de Dados e pela Comissão de Ética do Centro Hospitalar em causa, com os respetivos números: 11816/2016 e CE-no 27/16.

Os estudantes foram divididos em dois grupos: O Grupo de Controlo (GC), formado pelos estudantes que cumpriram o plano de ensino clínico na íntegra de acordo com o plano de estudos do curso de licenciatura em enfermagem e o Grupo de Teste (GT), composto pelos estudantes que, para além do cumprimento do plano de estudo, participaram da intervenção psicoeducativa dirigida ao estigma, desenhada para o contexto e público-alvo.

Os momentos de recolha de informação decorreram antes do início do ensino clínico e no último dia da sua realização. Os instrumentos foram aplicados por assistentes de investigação independentes. $\mathrm{O}$ seu preenchimento decorreu em sala própria com condiçóes de privacidade e anonimato.

Para a recolha dos dados foram utilizados quatro instrumentos, aplicados nas duas fases da pesquisa. O primeiro questionário foi composto por questóes sociodemográficas (idade, género, estado civil, com quem vive), clínicas. Incluíram-se ainda as questôes relativas à perceção do conhecimento e do estigma face à doença mental $(0=$ nenhum; $10=$ excelente / Total). O segundo instrumento foi constituído pela versão portuguesa da Mental Illness: Clinicians' Attitudes Scale (MICA-4) para determinação do estigma dos estudantes de saúde relativamente à doença mental. É um instrumento constituído por 16 itens numa escala do tipo Lickert, com scores de 1 - Concordo fortemente a 6 - Discordo fortemente, ${ }^{(18)}$ variando a escala de 16 a 96 pontos, onde pontuaçóes mais elevadas correspondem a atitudes mais estigmatizantes. O terceiro instrumento consistiu na versão portuguesa da Attribution Questionnaire $(\mathrm{AQ}-27)^{(19,20)}$ para avaliar o estigma social e as atitudes estigmatizantes dos estudantes. A AQ-27 é composta por uma vinheta clínica sobre saúde mental e 27 itens com respostas do tipo Lickert (1 a 9) caracterizando o estigma em nove dimensóes: Responsabilidade, Pena, Irritação, Perigosidade, Medo, Ajuda, Coação, Segregação, Evitamento. ${ }^{(20)}$ 
Pontuaçóes mais altas correspondem a maior estigma face à pessoa com doença mental e cada uma das dimensóes da AQ-27 varia entre 3 e 27 pontos. A Intergroup Anxiety Scale (EAI), escala de Ansiedade intergrupal foi o quarto instrumento utilizado para avaliar a ansiedade e experimentada pelos estudantes nas relaçóes grupais com as pessoas com doença mental e as suas implicações nas relaçôes através do autorrelato. ${ }^{(21)}$ A escala apresenta 12 itens que refletem diferentes estados de ansiedade nas componentes afetiva, cognitiva e comportamental que são cotados de 0 a 4 ( $0=$ nada a $4=$ extremamente). A pontuação das emoçôes positivas foi revertida para efeitos do score final (0 a 48 pontos); pontuações mais altas correspondem a níveis mais elevados de ansiedade intergrupal. ${ }^{(21)}$

O programa psicoeducativo para os estudantes de enfermagem resultou do benchmarking da intervenção desenvolvida no âmbito do projeto INDIGO liderada pelo Professor Doutor Graham Thornicroft do King's College of London. ${ }^{(22)}$ Tomando como referência a intervenção $R E A D$, adaptou-se à cultura da instituição, considerando os resultados da investigação prévia realizada com estudantes de enfermagem. ${ }^{(23,24)} \mathrm{O}$ planeamento da intervenção teve a autorização e a supervisão científica da Professora Doutora Claire Henderson. O programa de intervenção foi desenvolvido pelo investigador com formação especializada em enfermagem de saúde mental e psiquiátrica em três sessôes semanais. A primeira sessão focou-se na abordagem de conceitos, e abordagem da temática do estigma e os profissionais de saúde. A segunda sessão foi dedicada à identificação, reconhecimento e atuação perante situaçôes estigmatizantes e as suas consequências. A última sessão foi dedicada à reflexão, debate e discussão das medidas de ação perante situaçóes estigmatizantes. As estratégias pedagógicas incluíram exposição de conteúdos, trabalho de campo, discussão em grupo, role-play, análise e discussão de vídeos. Em todas as sessóes foi estimulada a participação ativa dos estudantes permitindo a expressão de sentimentos, dúvidas e preocupaçóes.

Para a análise dos dados foi usado o programa SPSS 24.0. Foram realizadas análises descritivas, frequências absolutas e relativas para as variáveis nominais e medidas de posição (média) e de variabilidade (desvio padrão) para as variáveis contínuas.
Utilizaram-se testes paramétricos nos testes das hipóteses, assumindo a normalidade por aplicação do teorema do limite central: $t$ de Student para a comparação das variáveis entre os dois momentos de avaliação e o teste de correlação de Pearson para variáveis contínuas. O teste Mann-Whitney foi usado para analisar as diferenças em variáveis não contínuas. $\mathrm{O}$ nível de significância adotado foi de 0,05. ${ }^{(25,26)}$

\section{Resultados}

Da totalidade da amostra, 49 estudantes constituiram o grupo de teste e 50 fizeram parte do grupo de controlo. As características sociodemográficas de ambos os grupos não se diferenciam estatisticamente, excetuando a preocupação perante pessoas com doença mental, embora sem diferença na ansiedade intergrupal (Tabela 1).

Dos 99 estudantes de enfermagem da amostra, $88(88,9 \%)$ eram do género feminino, com idades entre 20 e 40 anos (média=21,98, $\pm 3,414$ ), em grande maioria solteiros $(96 ; 97,0 \%)$.

$\mathrm{Na}$ avaliação inicial, os estudantes apresentavam em média uma baixa autoperceção de estigma (3,01 $\pm 1,776$ ), com valores médios / baixos de estigma quando avaliados com a MICA-4-PT (35,52 \pm $6,899)$. A ajuda e a pena foram as atitudes reveladoras de maior estigma, seguidas da coação (tabela 1). Em termos de ansiedade intergrupal, os resultados são satisfatórios $(2,22 \pm 0,521)$. A estranheza $(1,17$, $\pm 0,915)$ e apreensão $(1,35, \pm 0,719)$ foram pouco referidos, sendo a despreocupação $(3,41, \pm 0,833)$ e a descontração $(3,28, \pm 0,821)$ os sentimentos que mais contribuiram para a anisedade intergrupal dos estudantes.

Por forma a testar a hipótese 1 , analisou-se a diferença entre os dois momentos de avaliação, através do teste $t$ para amostras emparelhadas, comparando as médias das variáveis em análise nos dois momentos em ambos os grupos (Tabela 2). Verificou-se uma diminuição significativa do estigma autopercecionado e medido pela MICA-4 em ambos os grupos. Foram exceçôes as dimensôes de ajuda e coação que aumentaram as médias no segundo momento, estatisticamente significativas no grupo de teste. Foi 
Tabela 1. Caracterização do grupo de controlo e de teste no momento inicial (antes da intervenção)

\begin{tabular}{|c|c|c|c|c|c|c|c|c|c|}
\hline & & \multirow{2}{*}{\multicolumn{2}{|c|}{$\begin{array}{c}\text { Amostra total } \\
\mathrm{n}(\%)\end{array}$}} & \multirow{2}{*}{\multicolumn{2}{|c|}{$\begin{array}{c}\text { Grupo de teste } \\
\mathrm{n}(\%)\end{array}$}} & \multirow{2}{*}{\multicolumn{2}{|c|}{$\begin{array}{c}\text { Grupo de controlo } \\
n(\%)\end{array}$}} & \multicolumn{2}{|c|}{ Diferença entre grupos } \\
\hline & & & & & & & & $U^{*}$ & $p$-value \\
\hline \multirow[t]{2}{*}{ Género } & Feminino & \multicolumn{2}{|c|}{$88(88,9)$} & \multicolumn{2}{|c|}{$42(85,7)$} & \multicolumn{2}{|c|}{$46(92,0)$} & $-0,990$ & 0,326 \\
\hline & Masculino & \multicolumn{2}{|c|}{$11(11,1)$} & \multicolumn{2}{|c|}{$7(14,3)$} & \multicolumn{2}{|c|}{$4(8,0)$} & & \\
\hline \multirow[t]{2}{*}{ Estado civil } & Solteiro & \multicolumn{2}{|c|}{$96(97,0)$} & \multicolumn{2}{|c|}{$47(95,9)$} & \multicolumn{2}{|c|}{$49(98,0)$} & $-0,601$ & 0,548 \\
\hline & Casado / União de facto & \multicolumn{2}{|c|}{$3(3,0)$} & \multicolumn{2}{|c|}{$2(4,1)$} & \multicolumn{2}{|c|}{$1(2,0)$} & & \\
\hline \multirow[t]{3}{*}{ Sofrer de doença mental } & Sim & \multicolumn{2}{|c|}{$20(6,9)$} & \multicolumn{2}{|c|}{$7(14,3)$} & \multicolumn{2}{|c|}{$7(14)$} & $-0,41$ & 0,968 \\
\hline & Não & \multicolumn{2}{|c|}{$235(81,6)$} & \multicolumn{2}{|c|}{$42(85,7)$} & \multicolumn{2}{|c|}{$43(86)$} & & \\
\hline & & Média & $\begin{array}{l}\text { Desvio } \\
\text { padrão }\end{array}$ & Média & $\begin{array}{l}\text { Desvio } \\
\text { padrão }\end{array}$ & Média & $\begin{array}{l}\text { Desvio } \\
\text { padrão }\end{array}$ & $\mathrm{t}^{\star}$ & $\mathrm{p}$-value \\
\hline \multicolumn{2}{|l|}{ Idade } & 21,98 & 3,414 & 22,43 & 4,005 & 21,54 & 2,682 & 1,299 & 0,197 \\
\hline \multicolumn{2}{|l|}{ Perceção do nível de estigma } & 3,01 & 1,776 & 2,47 & 1,515 & 2,38 & 1,602 & 0,285 & 0,776 \\
\hline \multicolumn{2}{|c|}{ Estigma em saúde mental (MICA-4) } & 32,52 & 6,899 & 32,37 & 6,963 & 32,66 & 6,903 & $-0,210$ & 0,834 \\
\hline \multirow{9}{*}{$\begin{array}{l}\text { Estigma em saúde mental } \\
\text { (AQ-27) }\end{array}$} & Responsabilidade & 8,78 & 3,086 & 8,51 & 3,130 & 9,04 & 3,050 & $-0,853$ & 0,396 \\
\hline & Pena & 14,70 & 4,925 & 14,55 & 5,120 & 14,84 & 4,774 & $-0,291$ & 0,772 \\
\hline & Irritação & 7,35 & 3,541 & 7,49 & 3,489 & 7,22 & 3,621 & 0,377 & 0,707 \\
\hline & Perigosidade & 8,45 & 4,074 & 8,10 & 3,687 & 8,80 & 4,431 & $-0,851$ & 0,397 \\
\hline & Medo & 8,45 & 4,381 & 8,20 & 4,238 & 8,70 & 4,546 & $-0,561$ & 0,576 \\
\hline & Ajuda & 21,95 & 4,441 & 22,37 & 4,091 & 21,54 & 4,765 & 0,926 & 0,357 \\
\hline & Coação & 13,68 & 3,719 & 13,65 & 3,655 & 13,70 & 3,819 & $-0,062$ & 0,950 \\
\hline & Segregação & 6,58 & 3,637 & 6,08 & 3,297 & 7,06 & 3,914 & $-1,344$ & 0,182 \\
\hline & Evitamento & 10,63 & 5,585 & 10,69 & 5,459 & 10,56 & 5,761 & 0,119 & 0,906 \\
\hline Ansiedade intergrupal & TOTAL & 2,22 & 0,521 & 2,16 & 0,523 & 2,27 & 0,518 & $-0,947$ & 0,326 \\
\hline
\end{tabular}

Tabela 2. Estatística descritiva e Teste t para comparação do estigma e ansiedade intergrupal antes a após a intervenção em cada um dos grupos de teste e de controlo

\begin{tabular}{|c|c|c|c|c|c|c|c|c|c|}
\hline & & \multicolumn{4}{|c|}{ Grupo de Teste } & \multicolumn{4}{|c|}{ Grupo de Controlo } \\
\hline & & $\begin{array}{c}\text { Antes } \\
\text { Média (DP) }\end{array}$ & $\begin{array}{c}\text { Após } \\
\text { Média (DP) }\end{array}$ & $r$ & $\begin{array}{c}t \text { de Student/ } \\
p \text {-value }\end{array}$ & $\begin{array}{c}\text { Antes } \\
\text { Média (DP) }\end{array}$ & $\begin{array}{c}\text { Após } \\
\text { Média (DP) }\end{array}$ & $r$ & $\begin{array}{c}t \text { de Student/ } \\
p \text {-value }\end{array}$ \\
\hline \multicolumn{2}{|c|}{ Estigma autopercecionado } & $3,04(1,859)$ & $2,47(1,515)$ & 0,296 & $1,979^{\star}$ & $2,98(1,708)$ & $2,38(1,602)$ & 0,406 & $2,348^{\star \star}$ \\
\hline \multicolumn{2}{|c|}{ Estigma (MICA-4-PT) } & $32,37(6,963)$ & $29,23(7,121)$ & 0,707 & $3,711^{\star \star}$ & $32,66(6,903)$ & $29,02(6,951)$ & 0,720 & $4,896^{\star \star}$ \\
\hline \multirow{9}{*}{$\begin{array}{l}\text { Estigma em } \\
\text { saúde mental } \\
\text { (AQ-27) }\end{array}$} & Responsabilidade & $8,51(3,130)$ & $8,73(3,438)$ & 0,346 & $-0,417$ & $9,04(3,050)$ & $8,36(2,678)$ & 0,455 & 1,599 \\
\hline & Pena & $14,55(5,120)$ & $13,42(4,188)$ & 0,606 & 1,864 & $14,84(4,774)$ & $12,90(4,953)$ & 0,332 & $2,440^{\star \star}$ \\
\hline & Irritação & $7,49(3,489)$ & $6,16(2,771)$ & 0,336 & $2,541^{\star \star}$ & $7,22(3,621)$ & $6,06(3,621)$ & 0,588 & $2,619^{\star \star}$ \\
\hline & Perigosidade & $8,10(3,687)$ & $5,77(2,702)$ & 0,421 & $4,603^{\star \star}$ & $8,80(4,431)$ & $6,24(3,388)$ & 0,652 & $5,036^{\star \star}$ \\
\hline & Medo & $8,20(4,238)$ & $5,24(2,250)$ & 0,263 & $4,88^{\star \star}$ & $8,70(4,546)$ & $6,63(3,844)$ & 0,634 & $4,009^{\star \star}$ \\
\hline & Ajuda & $22,27(4,077)$ & $23,75(2,964)$ & 0,409 & $-2,600^{\star \star}$ & $21,54(4,765)$ & $22,80(4,005)$ & 0,425 & $-1,103$ \\
\hline & Coação & $13,65(3,655)$ & $15,38(3,598)$ & 0,310 & $-2,850^{\star \star}$ & $13,70(3,819)$ & $14,40(3,953)$ & 0,324 & $-1,095$ \\
\hline & Segregação & $6,08(3,297)$ & $5,48(2,821)$ & 0,623 & 1,539 & $7,06(3,919)$ & $5,52(3,221)$ & 0,608 & $3,381^{\star \star}$ \\
\hline & Evitamento & $10,69(5,459)$ & $8,24(2,250)$ & 0,633 & $4,036^{\star \star}$ & $10,56(5,761)$ & $9,02(5,285)$ & 0,683 & $2,508^{\star \star}$ \\
\hline \multirow{13}{*}{$\begin{array}{l}\text { Ansiedade } \\
\text { intergrupal }\end{array}$} & Ansiedade & $1,55(0,765)$ & $0,82(0,667)$ & 0,080 & $5,279^{\star \star}$ & $1,68(0,978)$ & $1,00(0,808)$ & 0,026 & $3,839^{\star \star}$ \\
\hline & Apreensão & $1,35(0,663)$ & $0,80(0,645)$ & 0,315 & $5,039^{\star \star}$ & $1,36(0,776)$ & $0,82(0,825)$ & 0,358 & $4,204^{\star \star}$ \\
\hline & Conforto & $2,88(0,881)$ & $1,97(1,010)$ & 0,278 & $5,509^{\star \star}$ & $3,06(0,682)$ & $2,10(1,09)$ & 0,183 & $5,766^{\star \star}$ \\
\hline & Segurança & $2,78(0,798)$ & $1,97(0,923)$ & 0,220 & $5,160^{\star \star}$ & $2,78(0,815)$ & $1,98(1,059)$ & 0,349 & $5,199^{\star \star}$ \\
\hline & Preocupação & $1,37(1,014)$ & $0,67(0,718)$ & 0,254 & $4,481^{\star \star}$ & $1,60(0,782)$ & $0,760(0,687)$ & 0,046 & $5,838^{\star \star}$ \\
\hline & Calma & $2,86(0,646)$ & $1,95(1,117)$ & 0,078 & $5,046^{\star \star}$ & $2,76(0,744)$ & $1,96(1,194)$ & 0,196 & $4,427^{\star \star}$ \\
\hline & Confiança & $2,73(0,638)$ & $2,06(1,008)$ & 0,123 & $4,190^{\star \star}$ & $2,92(0,778)$ & $2,22(1,035)$ & 0,174 & $4,186^{\star \star}$ \\
\hline & Estranheza & $1,20(0,841)$ & $0,50(0,619)$ & 0,486 & $6,68^{\star \star}$ & $1,14(0,990)$ & $0,90(0,863)$ & 0,088 & 1,353 \\
\hline & Tensão & $1,41(0,840)$ & $0,59(0,537)$ & 0,239 & $6,478^{\star \star}$ & $1,34(0,872)$ & $0,820(0,825)$ & 0,172 & $3,366^{\star \star}$ \\
\hline & Despreocupação & $3,22(0,919)$ & $2,55(1,225)$ & 0,258 & $3,547^{\star \star}$ & $3,60(0,700)$ & $2,56(1,387)$ & 0,193 & $0,515^{\star \star}$ \\
\hline & Nervosismo & $1,43(0,890)$ & $0,80(0,612)$ & 0,164 & $4,457^{\star \star}$ & $1,58(0,835)$ & $1,04(0,856)$ & 0,167 & $3,497^{\star \star}$ \\
\hline & Descontração & $3,18(0,808)$ & $2,32(1,214)$ & 0,362 & $5,041^{\star \star}$ & $3,38(0,830)$ & $2,32(1,202)$ & 0,182 & $5,631^{\star \star}$ \\
\hline & TOTAL & $2,16(0,523)$ & $1,40(0,579)$ & 0,292 & $7,889^{* *}$ & $2,27(0,518)$ & $1,54(0,735)$ & 0,110 & $6,033^{\star \star}$ \\
\hline
\end{tabular}

(DP)- Desvio Padrão, * $p$-value $<0,05 ;{ }^{* *} p$-value $<0,01$

a dimensão do medo (Diferença de média=2,959)

e perigosidade que revelou maiores diferenças de médias entre o primeiro e o segundo momento, so- bretudo no grupo de teste. No segundo momento verificou-se uma redução dos níveis de ansiedade intergrupal dos estudantes perante o doente mental, 
que foram significativas para todos os sentimentos dos estudantes do grupo de teste.

Na tabela 3 apresentam-se os resultados do teste da hipótese 2. A significância da diferença entre o estigma e a ansiedade dos estudantes sujeitos à intervenção dirigida ao estigma comparativamente aos que não estiveram sujeitos à intervenção foi avaliada pelo teste $t$ de student para amostras independentes. Observaram-se valores estatisticamente idênticos de estigma autopercecionado, avaliado pela MICA-4 e ansiedade intergrupal entre os grupos de teste e de controlo, pelo que não comprovamos a hipótese. Não obstante, observou-se uma diminuição significativa do estigma no grupo de teste relativo ao medo comparativamente ao grupo de controlo. Inversamente verificou-se um aumento do estigma na dimensão da ajuda em ambos os grupos sendo maior no grupo de teste $(1,47, \pm 0,714)$ (Tabela 3 ).

Tabela 3. Resultados da aplicação do teste t de student para determinação das diferenças de médias entre 0 grupo de teste e 0 grupo de controlo no $2^{\circ}$ momento

\begin{tabular}{llccccc}
\hline & $\begin{array}{c}\text { Diferença } \\
\text { média }\end{array}$ & $\begin{array}{c}\text { Erro padrão } \\
\text { da diferença }\end{array}$ & $\boldsymbol{t}$ & $\boldsymbol{p}$-value \\
\hline Estigma autopercecionado & 0,061 & 0,359 & 0,170 & 0,866 \\
\multicolumn{2}{c|}{ Estigma (MICA-4-PT) } & 0,217 & 1,459 & 0,149 & 0,882 \\
Estigma em & Responsabilidade & 0,374 & 0,618 & 0,606 & 0,546 \\
saúde mental & Pena & 0,528 & 0,922 & 0,573 & 0,568 \\
(AQ-27) & Irritação & 0,103 & 0,605 & 0,170 & 0,865 \\
& Perigosidade & $-0,469$ & 0,619 & $-0,758$ & 0,450 \\
& Medo & $-1,387$ & 0,636 & $-2,181$ & $0,032^{*}$ \\
& Ajuda & 1,470 & 0,714 & 2,071 & $0,041^{*}$ \\
& Coação & 0,987 & 0,760 & 1,299 & 0,197 \\
& Segregação & 0,961 & $-0,030$ & $-0,50$ & 0,961 \\
Ansiedade intergrupal & Evitamento & $-0,775$ & 0,935 & $-0,829$ & 0,409 \\
\hline
\end{tabular}

\section{Discussão}

As características sociodemográficas dos estudantes foram semelhantes a outros estudos, como a maioria serem mulheres, jovens adultas e solteiras. $(1,2,7,14)$ Os valores de estigma observados na amostra estão em linha com os estudos realizados com os estudantes em diferentes contextos. ${ }^{(1,7,10,13)}$ No segundo momento do estudo verificou-se uma diminuição tanto no grupo de teste como no grupo de controlo. Parte destes resultados corroboram os de outras investigaçóes, podendo ser explicados pelo contacto dos estudantes com as pessoas com doença mental e com os profissionais. ${ }^{(1,7,-11-15)} \mathrm{O}$ ensino clínico em enfermagem de saúde mental e psiquiatria contribuíu para uma redução das atitudes negativas no geral, afetando positivamente e de forma significativa as perceçóes e atitudes dos estudantes face à pessoa com doença mental, corroborando os resultados de outras pesquisas. ${ }^{(1,11-15)}$ De facto, os cursos de bacharelato em enfermagem em Portugal compreendem uma parte teórico-prática de lecionação de conteúdos dirigidos à doença mental e suas implucaçôes nos doentes e famílias, realizada em contexto escolar, e uma parte prática de ensino clínico realizada em ambientes reais na presença de pessoas com doença mental e suas famílias. A revisão sistemática realizada sobre as atitudes face à doença mental dos estudantes da formação inicial de enfermagem demonstra uma tendência de atitudes mais favoráveis nos que tiveram mais horas de preparação teórica e ensinos clínicos mais longos. ${ }^{(27,28)}$ Ainda que não seja possível a comparação com outros estudantes de outras escolas portuguesas, a composição mista do curriculum do curso dos estudantes da nossa amostra bem como a duração do ensino teórico e prática clínica (das mais longas do ensino em Portugal) pode explicar os resultados em linha com a literatura. ${ }^{(28,29)}$

Por outro lado, constatou-se um aumento dos estereótipos relativos à ajuda e coação o que pode indicar uma atitude paternalista subjacente ao cuidado, marcada por um sentimento de pena e consequente aumento da controlabilidade. ${ }^{(19)}$ Estes resultados podem ser parcialmente explicados pela influência do contexto onde decorreu o ensino clínico (serviços de "porta fechada") e pela atitude dos enfermeiros no desempenho do papel de supervisores de estudantes de enfermagem. ${ }^{(13)}$ Em Portugal, em linha com as orientaçôes internacionais, tem-se apostado numa mudança dos cuidados na doença mental mais abertos e centrados na comunidade, em que os enfermeiros têm um papel fundamental na mudança desses cuidados. Não obstante, os relatórios de execução do Plano Nacional de Saúde Mental referem um défice do número de enfermeiros alocados às equipas multidisciplinares o que dificulta a adoção de novas abordagens centradas na pessoa doente e menos paternalistas. ${ }^{(30)}$ Apesar da composição e duração do 
currículo dos estudantes, a influência dos contextos clínicos, ainda estruturados nos modelos mais tradicionais podem ter reforçado a cultura de estigmatização socialmente enraizada.

A intervenção dirigida ao estigma não demonstrou efeito diferenciador da ansiedade intergrupal nem nas atitudes estigmatizantes face à pessoa com doença mental a não ser no medo (com redução significativa no grupo de teste) e na ajuda (aumento significativo), sugerindo a necessidade da sua reformulação. Alguns autores defendem a necessidade de se trabalhar com os estudantes aspetos de desenvolvimento pessoal, atitudes na componente do cuidado, habilidades comunicacionais e relacionais e competências que lhe permitam identificar as sua próprias crenças e atitudes negativas em relação a pessoas com doença mental. ${ }^{(14)}$

O presente estudo apresenta algumas limitaçóes que condicionam a interpretação e extrapolação dos resultados. A técnica de amostragem e o reduzido tamanho da amostra constituem uma limitação à generalização dos resultados. Por outro lado, o desenho do estudo designou o grupo de teste para realização do ensino clínico num hospital diferente do grupo de controlo. A diferença de contextos de ensino clínico e a cultura inerente aos diferentes serviços pode ter condicionado os níveis de estigma e de ansiedade intergrupal e mascarado os possíveis efeitos da intervenção. Esta limitação tem implicaçóes na pesquisa, apontando para a necessidade de reformulação do desenho do estudo, garantindo que o grupo de teste e de controlo são sujeitos às mesmas condições de ensino clínico. Outra limitação pode ter sido relativa ao dinamizador das sessóes do programa de intervençáo, que no presente estudo era previamente conhecido pelos estudantes e teve contacto prévio com os mesmos durante a formação teórica. Pensamos que outros resultados poderiam ser obtidos se o interventor pertencesse ao grupo de enfermeiros especializados dos serviços de psiquiatria e não tivesse contactado previamente com os estudantes.

$\mathrm{O}$ presente estudo contribuiu para o aumento do conhecimento do impacto de um programa de intervenção de três dias realizado em contexto de ensino clínico na ansiedade intergrupal e no estigma dos estudantes portugueses. Apesar dos resultados náo apontarem para o efeito desejado do programa, reconhecemos implicaçóes destes na Enfermagem ao nível da prática clínica, ensino, gestão e investigação.

Ao nível da prática clínica o estudo aponta para a necessidade do envolvimento ativo dos profissionais dos serviços na programação e implementação de programas de intervenção dirigidos ao estigma dos estudantes em ensino clínico. Quanto ao ensino, sugere-se a utilização de métodos pedagógicos alternativos com vista à redução do estigma de forma integrada, com maior ênfase na temática desde os primeiros anos do curso. Por outro lado, a abordagem dos conteúdos de enfermagem de saúde mental e psiquiátrica está focada no ensino dos sinais, sintomas e intervençôes terapêuticas dirigidas às pessoas com doença mental e seus cuidadores. Pouco tempo letivo se dedica aos estudantes para que reflitam sobre as suas atitudes e sentimentos. Neste sentido, urge proporcionar espaços de reflexão e debate de ideias face à doença mental em contexto académico. Os resultados também apontam para a necessidade de uma mudança curricular que espelhe a centralidade no cliente e a parceria com o mesmo na prestação de cuidados, com vista à redução do estigma relacionado com a ajuda e coação. Uma reformulação dos curricula, apostando no envolvimento dos estudantes na co-construção de programas anti-estigma inovadores, que incluam maior proximidade com as pessoas com doença mental em contextos abertos, num modelo de workshops residenciais poderiam ser promissores na erradicação do estigma. A nível da gestão sugere-se uma maior inclusão da pessoa com doença mental no planeamento dos seus cuidados em contextos de internamento hospitalar à semelhança do que é preconizado nos modelos de recovery. A inclusão dos estudantes em contextos desta natureza durante o ensino clínico teria impacto expectável na forma como se relacionam com os clientes com doença mental e na redução do próprio estigma. Novas pesquisas são essenciais para identificar os modelos e estratégias de ensino/ aprendizagem, dirigidas às populaçóes dos estudantes, professores, profissionais de saúde, doentes e cuidadores no sentido de mitigar as perceçôes negativas face à doença mental. 


\section{Conclusão}

Partindo do objetivo traçado para esta investigação, constata-se que o ensino clínico promove uma redução das atitudes estigmatizantes e ansiedade perante o doente mental dos estudantes de enfermagem. A intervenção dirigida ao estigma não revelou efeitos significativos no estigma global nem na ansiedade intergrupal o que aponta para a necessidade futura da sua reformulação e comparação com amostras de estudantes de outras instituiçóes de ensino de enfermagem. Sendo este estudo realizado apenas numa instituição de ensino de Enfermagem, os resultados devem ser interpretados com cautela, bem como as conclusóes que dele resultam, na medida em que a amostra não é representativa da população portuguesa.

\section{Colaborações}

Querido AIF, Tomás CC, Carvalho DRS, Gomes JMF e Cordenrio MSS contribuíram com a conepção do projeto, análise e interpretação dos dados, redação do artigo, revisão crítica relavante do conteúdo intelectual e aprovação da versão final a ser publicada.

\section{Referências}

1. Gil IM, Santos JC, Loureiro LM. [Stigma in Nursing Students: Before and After Contact with People with Mental Disorders]. Rev Enferm UERJ. 2016; 24 (1): 1-7. Portuguese.

2. Ferreira, FN, Fernandino, DC, Souza, GR, Ibrahim, TF, Fukino, AS, Araújo, NC, et al. [Assessment of Health Students Attitudes towards Schizophrenic Patients]. Rev Bras Educ Méd. 2015; 39(4): 42-9. Portuguese.

3. Abramenko L, Lovisi GM, Fonseca DL, Abelha L. [Attitudes of mental health workers towards psychiatric patients in a city in the interior of Rio de Janeiro State]. Cad. Saúde Colet. 2017; 25(2):169-76. Portuguese.

4. Fukuda CC, Penso MA, Amparo DM, Almeida BC, Morais, CA. Mental health of young Brazilians: Barriers to professional help-seeking. Estud Psicol. 2017; 33 (2):355-65.

5. Clemente As, Santos WJ, Nicolato R, Firmo JO. Stigma related to bipolar disorder in the perception of psychiatrists from Belo Horizonte, Minas Gerais State, Brazil. Cad Saúde Pública. 2017; 33(6):e00050016.

6. Baptista, MN, Zanon, C. Why not Seek Therapy? The Role of Stigma and Psychological Symptoms in College Students. Paidéia (Ribeirão Preto). 2017; 27 (67):76-83.
7. Duman ZC, Günüșen NP, İnan FS, Ince SC, Sari A. Effects of Two Different Psychiatric Nursing Courses on Nursing Students' Attitudes Towards Mental IIIness, Perceptions of Psychiatric Nursing, and Career Choices. J Prof Nurs. 2017; 33 (6):452-9.

8. De Witt C, Smit I, Jordaan E, Koen L, Niehaus DJ, Botha U. The impact of a psychiatry clinical rotation on the attitude of South African final year medical students towards mental illness. BMC Med Educ. 2019; $19(1): 114-8$.

9. Lauber C, Anthony M, Ajdacic-Gross V, Rössler W. Evaluating explicit and implicit estigma on mental illness in mental health professionals and medical students. Community Ment Health J. 2015; 51(5):628-34.

10. Grandón P, Aguilera AV, Bustos C, Alzate EC, Saldivia S. [Evaluation of stigma towards people diagnosed with schizophrenia through a knowledge]. Rev Colomb Psiquiatr. 2018; 47(2):72-81. Spanish.

11. Gulati P, Das S, Chavan BS. Impact of psychiatry training on attitude of medical students toward mental illness and psychiatry. Ind J Psychiatry. 2014 56(3):271-7.

12. Ahuja KK, Dhillon M, Juneja A, Sharma B. Breaking barriers: An education and contact intervention to reduce mental illness stigma among Indian college students. Psychosoc Intervent. 2017;26(2):1039

13. López LP, Rebolledo NO, Ahumada CM. [Effects of clinical experience on the attitudes of nursing students towards people with psychiatric disabilities]. Cienc Enferm. 2017; 23(3):125-32. Spanish.

14. Granados-Gámez G, López MD, Corral GU, Márquez-Hernández VV. Attitudes and beliefs of nursing students toward mental disorder: The significance of direct experience with patients. Perspect Psiquiatr Care. 2017; 53(2):135-43.

15. Estévez MC, Guinón FG, raposo IF, Víchez EM. [The face of madness. Attitude of the nursing nurses towards the mental disorder]. Agora de Enfermaria. $2017 ; 21(2): 61-5$. Spanish.

16. Thornicroft G, Mehta N, Clement S, Evans-Lacko S, Doherty M, Rose D, Henderson C. Evidence for effective interventions to reduce mental-health-related stigma and discrimination. 2016. The Lancet, 387(10023): 1123-32.

17. Mehta N, Clement S, Marcus E, Stona A-C, Bezborodovs $N$, Evans-Lacko $S$, et al. Systematic review of evidence for effective interventions to reduce mental health related stigma and discrimination: medium and long-term effectiveness and interventions in low- and middle-income countries. Br J Psychiatry. 2015;207 (5):377-84.

18. Gabbidon J, Clement S, van Nieuwenhuizen A, Kassam A, Brohan E, Norman I, et al. Mental IIIness: Clinicians' Attitudes (MICA) ScalePsychometric properties of a version for healthcare students and professionals. Psychiatry Res. 2013;206(1):81-7.

19. Corrigan P, Markowitz F, Watson A, Rowan D, Kubiak MA. An attribution model of public discrimination towards persons with mental illness. J Health Soc Behav. 2003;44:162-79.

20. Sousa S, Queirós C, Marques A, Rocha N, Fernandes A. Versão preliminar portuguesa do Attribution Questionnaire (AQ-27). [adaptada com autorização de P. Corrigan]. Porto: FPCEUP/ ESTSPIPP; 2008.

21. Stephan W, Stephan C. Intergroup anxiety. J Soc Issues. 1985, 41(3):157-75.

22. Centre for Global Mental Health. The INDIGO Network [Internet]. London: London School of Hygiene and Mental Health, Kings College; 2018. [cited 2019 Jun 1]. Available from: https://www. centreforglobalmentalhealth.org/the-indigo-network 
23. Winkler $P$, Janousková M, Kozený J, Pasz J, Mladá K, Weissová A, Tusková E, Evans-Lacko $S$. Short video interventions to reduce mental health stigma: a multicentre randomised controlled trial in nursing high schools. Soc Psychiatry Psychiatr Epidemiol (2017) 52:1549-57.

24. Querido A, Tomás C, Carvalho D. 0 Estigma face à doença mental nos estudantes de saúde. Rev Port Enferm Saúde Mental. 2016;(Spe 3): 67-72. Portuguese.

25. Pestana M, Gageiro J. Análise de dados para ciências sociais. A complementaridade do SPSS. 6a ed. Lisboa; Edições Sílabo; 2014.

26. Marôco J. Análise estatística com o SPSS Statistics. 7a ed. Pero Pinheiro: Report Number; 2018.

27. Happell B, Platania-Phung C, Bocking J, Scholz B, Horgan A, Manning F, et al. (2018). Nursing Students' Attitudes Towards People Diagnosed with Mental IIIness and Mental Health Nursing: An International Project from Europe and Australia. Iss Mental Health Nurs. 2018;39(10):82939.

28. Happell B, Gaskin CJ. The attitudes of undergraduate nursing students towards mental health nursing: A systematic review. J Clin Nurs. 2013;22(1-2):148-58.

29. Sherwood DA. Healthcare curriculum influences on stigma towards mental illness: Core psychiatry course impact on pharmacy, nursing and social work student attitudes. Cur Pharm Teach Learn. 2019;11(2):198-203.

30. Xavier M, Paixão I, Mateus P, Goldschmidt T, Pires P, Narigão M, et al., coordenadores. Relatório da Avaliação do Plano Nacional de Saúde Mental 2007-2016 e propostas prioritárias para a extensão a 2020. República Portuguesa: Serviço Nacional de Saúde; 2017; p.1-62. 